\title{
Systemic Biomarkers for Periodontitis
}

\author{
Panagiota G. Stathopoulou ${ }^{1} \cdot$ Nurcan Buduneli $^{2} \cdot$ Denis F. Kinane ${ }^{3}$
}

Published online: 12 October 2015

(C) Springer International Publishing AG 2015

\begin{abstract}
Periodontitis affects a subset of the population and our current thinking is that progression of periodontal disease may be either continuous or episodic. These features make diagnostic tools for disease status and progression assessment desirable in the management of this disease. Although many potential markers exist, several difficulties hamper our ability to declare them diagnostic tests with proven utility. The "gold standard" for active periodontal disease is not available, and the best indicators currently available include clinical measurements with low sensitivity and specificity that are also time consuming in their assessment and recording. Although much is written about the need for markers of current or future disease which will prevent us from overtreating pockets, the time, effort, and cost involved in testing these sites have to be balanced against the relative ease and speed of routine nonsurgical periodontal therapy. In addition, we are still some way from the development and validation of reliable host factor/ microbial factor testing methods. There are, however, multiple local and systemic periodontal diagnostic biomarkers proposed within the literature, although none is presently widely available commercially. One could envisage chairside tests using saliva or blood from finger sticks being capable to determine an individual's risk of developing disease or a
\end{abstract}

This article is part of the Topical Collection on Epidemiology

Panagiota G. Stathopoulou

pstat@dental.upenn.edu

1 Department of Periodontics, School of Dental Medicine, University of Pennsylvania, Philadelphia, PA, USA

2 Department of Periodontology, School of Dentistry, Ege University, Izmir, Turkey

3 Departments of Pathology and Periodontics, School of Dental Medicine, University of Pennsylvania, Philadelphia, PA, USA patient's risk of disease progression and thus the need for a timely prevention program. In addition, blood drawn for routine diagnostic check-ups could also be used for the diagnosis and monitoring of periodontitis, if systemic biomarkers are identified. Before applying any test, we should reconsider what treatment planning effects a positive or negative result will have, since any test that does not influence the treatment plan is redundant. Systemic periodontal diagnostic tests are still at an early stage of development and much work remains to be performed to fully validate their utility such that they become an important and cost-effective aspect of clinical diagnosis, treatment planning, or patient monitoring. This paper provides a comprehensive review of systemic biomarkers derived form blood or saliva that have the potential to be utilized as a diagnostic and monitoring test for periodontitis at the patient level.

Keywords Biomarkers · Salivary markers $\cdot$ Periodontal diagnosis $\cdot$ Periodontitis screening

\section{Introduction}

The periodontal diseases are a family of chronic immune and inflammatory conditions of the periodontium that can be broadly divided into gingivitis, a superficial inflammation confined to the gingiva, and periodontitis, which is characterized by attachment loss and alveolar bone loss. Periodontitis, the destructive form of periodontal disease, affects almost $50 \%$ of American adults age 30 and older, and its prevalence increases with age [11]. Periodontal diseases, with the exception of a small group of non-plaque-induced gingival lesions [5], are initiated by microbial dental plaque and modified by local, systemic, and environmental factors [25]. It is estimated that over 700 different species are able to colonize the oral 
cavity [2], and at least 400 species can be found in subgingival plaque [37]. The level of the innate and immune response initiated by this variable dental plaque biofilm also varies and is highly depended upon the host susceptibility [24]. The clinical presentation of the resulting periodontal disease, disease severity, and disease progress is a summation of these complex host-bacterial interactions.

Currently, periodontitis is diagnosed almost entirely on the basis of an array of clinical measurements including clinical attachment level (CAL), bleeding on probing (BOP), probing depth (PD), and radiographic findings [1]. Additional information obtained by medical and family history, and specific characteristics of clinical presentation, such as quantity of local factors and location of lesions, are helpful in the differential diagnosis of specific types of periodontitis [5]. Accurate diagnosis at the patient and site level requires full mouth clinical measurements by means of a periodontal probe and recording multiple parameters at six sites per tooth, resulting in a laborious diagnostic process that is also dependent on the individual clinician performing the examination. Furthermore, this process needs to be repeated at regular intervals in order to determine the patient's disease status at recall visits. These clinical parameters are the best currently available indicators for determining disease status; however, they only provide information about past periodontal tissue destruction and do not elucidate current disease activity nor predict future activity due to low sensitivity and positive predictive value [19]. Therefore, one of the major challenges in the field of periodontics is to identify a quick, efficient, and objective diagnostic and monitoring method, with the ability to screen for susceptibility to periodontal disease, diagnose periodontal disease, evaluate response to treatment, predict future tissue destruction, and identify disease progression. The ideal diagnostic and monitoring method should be able to identify and monitor disease both at the patient and the site level.

Several molecules have been studied as potential biomarkers for periodontal disease including enzymes, cytokines, receptors, and other proteins. These biomarkers can be found in several biologic fluids, namely the gingival crevicular fluid (GCF) contains local biomarkers and can potentially provide information at the site level; the blood, serum, or plasma contain systemic biomarkers and can potentially provide information at the patient level; and saliva contains both local and systemically derived markers and provides information at the patient level as well. Although the GCF has the advantage that it provides information at the site level, GCF collection is rather complicated and more time-consuming than full mouth probing and thus not convenient during routine procedures in the dental office. On the other hand, saliva is a biological material that is abundant and the sampling procedure is easy, fast, non-invasive, and more convenient for the patient and clinician. Saliva cannot provide site-specific information; however, it is an easily accessible fluid, which contains local and systemically derived markers of periodontal disease [22]. Similarly, blood cannot provide site-specific information; its sampling is generally more invasive than that of saliva; however, it is easy, fast, and can be performed outside of the dental office and as part of a routine general diagnostic check-up; sampling may also be minimally invasive if performed via a finger stick. Because of the simple, fast, and relatively noninvasive method of collection, salivary and blood diagnostic tests have the potential to be used for diagnosis and monitoring of periodontal disease at the patient level.

The ideal diagnostic test should have high sensitivity, specificity, and predictive value. Sensitivity relates to the test's intrinsic ability to correctly detect patients who have a disease. Specificity relates to the test's intrinsic ability to correctly detect patients without a disease. The positive and negative predictive values are the proportions of positive and negative results in diagnostic tests that are true positive and true negative results. They describe the performance of a diagnostic test and are not intrinsic to the test but also depend on the prevalence of the disease. The positive predictive values can be derived using Bayes' theorem. Given that periodontitis is a disease with high prevalence of $50 \%$ in US adults older than 30 years, if a diagnostic test has high sensitivity and specificity of $99 \%$, then the positive predictive value, or the probability that a US patient older than 30 years who tests positive truly has periodontitis, is $99 \%$; the negative predictive value, or the probability that a patient older than 30 years who tests negative is truly periodontally healthy, is also $99 \%$. The same diagnostic test, with the same sensitivity and specificity, would have a higher positive predictive value and lower negative predictive value, in a population with higher prevalence of periodontitis, and a lower positive predictive value and higher negative predictive value, in a population with lower prevalence of periodontitis.

The aim of the present paper is to provide a comprehensive review of systemic biomarkers derived form blood or saliva that have the potential to be utilized as a diagnostic and monitoring test for periodontitis at the patient level.

\section{Biomarkers for Diagnosis of Diseased Individuals}

\section{Diagnosis—Blood Biomarkers}

Periodontitis is initiated by periodontal pathogens that elicit both an innate and humoral immune response; antibodies to some of these pathogens have been detected both locally at the gingival tissue as well as systemically in the blood stream. In order to determine if the levels of systemic antibodies to Porphyromonas gingivalis, the most researched periodontal pathogen, correlate with periodontal status, Trindade et al. evaluated patients with different types of periodontitis compared to healthy controls [44]. Serum IgG to fraction IV was 
statistically significantly higher in chronic periodontitis patients compared to healthy controls, while serum $\operatorname{IgG}, \operatorname{IgG} 2$, $\mathrm{IgG} 3$, and IgG4 to fraction IV was statistically significantly higher in aggressive periodontitis patients compared to healthy controls. This data suggest that serum IgG levels to $P$. gingivalis may be a useful systemic biomarker for diagnosis of both chronic and aggressive periodontitis.

Collectins are a family of pattern recognition receptors that are present in plasma and on mucosal surfaces that recognize several pathogen-associated molecular patterns (PAMPs), and are important in the innate immune response. Surfactant protein D (SP-D) is a collectin that recognizes surface glycoconjugates and lipopolysaccharide (LPS) of Gramnegative bacteria, and is widely expressed in epithelial cells of the digestive tract and in serum. In order to determine the correlation between periodontal disease and plasma SP-D, Glas et al. evaluated 105 chronic periodontitis patients and 122 healthy controls in a case-control study [16], and showed statistically significantly increased plasma SP-D concentrations in patients compared with controls that was independent of the patient's genotype. This data suggest that plasma SP-D may be a useful systemic biomarker for diagnosis of periodontal disease.

Several inflammatory biomarkers found in blood or saliva have been studied for their potential to be utilized in the diagnosis of periodontitis. In a case-control study, Nibali et al. evaluated 302 patients with severe periodontitis and 183 healthy controls to determine the correlation between periodontitis and inflammatory and metabolic biomarkers [34]. Leukocyte differential counts, high-density lipoprotein cholesterol (HDL), low-density lipoprotein cholesterol (LDL), and glucose were evaluated, and it was determined that there was an association between periodontitis and systemic inflammation and a dysmetabolic state. This data suggest that these commonly surveyed inflammatory and metabolic biomarkers may be promising systemic biomarkers for diagnosis of periodontitis in Caucasian patients who are systemically healthy and non-smokers.

C-reactive protein (CRP) is a circulating acute-phase protein acting in innate immune response. Significantly higher serum high-sensitivity CRP (hs-CRP) concentrations have been reported in periodontitis patients compared to healthy controls in various case-control studies $[8,10,12,17,27$, 28]. In addition, Nakajima et al. reported that both hsCRP and IL-6 were significantly higher in periodontitis patients compared to healthy controls [33]. These data suggest that serum hsCRP and IL-6 may be useful systemic biomarkers for diagnosis of periodontal disease.

In a cross-sectional study, Ishisaka et al. evaluated 467 elderly Japanese to determine the correlation between periodontal disease extent and cortisol [20]. Multiple regression analysis showed that a higher level of cortisol was significantly associated with greater numbers of sites with severe loss of
CAL only in non-smokers. This data suggest that serum cortisol may be a useful systemic biomarker for diagnosis of periodontal disease in non-smokers.

Osteocalcin (OC) is a non-collagenous Ca-binding protein of mineralized tissue, and the level of $\mathrm{OC}$ in serum is often used as a biomarker for bone turnover. In a cross-sectional study of 148 elderly Japanese, Yoshihara et al. showed that serum OC level was negatively associated with the percentage of sites with CAL loss of $6 \mathrm{~mm}$ or more, suggesting that serum OC may be a useful systemic biomarker for diagnosis of periodontal disease in this population [47]. Ozcaka et al. however, failed to find significant association between $\mathrm{OC}$ and periodontal status in a Turkish population [36]. Additional studies are needed in order to determine if serum $\mathrm{OC}$ can be a useful biomarker for other populations.

\section{Diagnosis-Salivary Biomarkers}

In a case-control study, Frodge et al. evaluated 35 patients with moderate to severe periodontitis and 39 healthy controls to determine the correlation between periodontitis and bone remodeling biomarkers in saliva [13]. Tumor necrosis factoralpha (TNF-alpha), C-telopeptide pyridinoline cross-links of type I collagen (ICTP), and receptor activator of nuclear factor-kappa B ligand (RANKL) were evaluated, and it was determined that salivary levels of TNF-alpha only were significantly higher in individuals with periodontal disease. Furthermore, patients with salivary TNF-alpha levels above a threshold of $5.75 \mathrm{pg} / \mathrm{ml}$ (i.e., two standard deviations above the mean of the controls) had significantly more sites with BOP, PD of $4 \mathrm{~mm}$ or more, and CAL loss $2 \mathrm{~mm}$ or more. This data suggest salivary TNF-alpha may be a useful systemic biomarker for diagnosis of periodontitis.

Matrix metalloproteinases (MMPs) are a superfamily of proteases with a role not only in physiological development and tissue remodeling but also in pathological tissue destruction [42]. MMPs are divided into five major groups: collagenases (MMP-1, MMP-8, MMP-13), gelatinases (MMP-2, MMP-9), stromelysins (MMP-3, MMP-10, MMP-11), membrane-type MMPs (MMP-14, MMP-15, MMP-16, MMP-17), and others. MMPs are produced by host cells including fibroblasts, macrophages, neutrophils, and epithelial cells. They collectively can degrade almost all components of extracellular matrix and basement membrane, and their excess activity can lead to periodontal tissue destruction; MMP activity is controlled by tissue inhibitors of MMPS (TIMPs). In a case-control study, Gursoy et al. evaluated salivary MMP-8, MMP-14, and TIMP-1 as potential biomarkers for periodontitis [18]; salivary MMP-8 and TIMP-1 concentrations were significantly higher in periodontitis patients compared to controls in non-smokers only; the MMP-8/TIMP-1 ratio, however, differentiated periodontitis and control groups even in smoker subjects. This data suggest that salivary MMP-8 and 
TIMP-1 are useful systemic biomarkers for diagnosis of periodontitis in non-smokers but not in smokers, while MMP-8/ TIMP-1 ratio may be promising as a biomarker for smokers as well.

In another case-control study, Miller et al. evaluated 28 patients with moderate to severe periodontitis and 29 healthy controls to determine if salivary IL-1beta and MMP- 8 could be used as potential biomarkers for periodontal disease, individually or combined [32]. Each biomarker was significantly higher in patients compared to healthy controls, while combined elevated salivary levels of IL-1beta and MMP-8 increased the risk of experiencing periodontal disease 45 -fold. This data suggest that salivary IL-1beta and MMP- 8 may be useful systemic biomarkers for diagnosis of periodontitis, and when evaluated in combination, they may increase the sensitivity of the diagnostic test.

The combined use of host- and bacteria-derived molecules as potential biomarkers was evaluated in a case-control study of 100 subjects by Ramseier et al. Patients with periodontitis exhibited significantly higher levels of salivary MMP-8 and MMP-9 [40]. Furthermore, the combination of salivary MMP8, MMP-9, and osteoprotegerin with red-complex anaerobic periodontal pathogens provided highly accurate predictions of periodontal disease category. This data further strengthen the position that a panel of biomarker signatures may be promising as a more accurate diagnostic test. The use of salivary MMP-8 as a potential biomarker for the diagnosis of periodontal disease has been validated in other studies [39], while additional systemic biomarkers found in blood or saliva have been identified as promising for the diagnosis of periodontal disease; these include oncostatin M, other MMPs, and salivary arginase activity; and these biomarkers may also be useful for monitoring disease progress and are reviewed in the following section.

\section{Biomarkers for Monitoring of Disease Progress}

Several inflammatory biomarkers found in blood or saliva have been studied for their potential to be utilized for monitoring the response to periodontal treatment; serum hsCRP, IL-6, oncostatin M, MMP-8 and MMP-9, and salivary sCD44, arginase, AST, ALT, LDH, sRANKL, and OPG have been identified as those with the greatest potential.

\section{Response to Treatment-Blood Biomarkers}

In an intervention study evaluating the effect of comprehensive periodontal therapy on 19 systemic inflammatory biomarkers using multiplex assays of plasma samples, Behle et al. found high variability between subjects and poor correlation between inflammatory markers in general and clinical, microbiological, and serological markers of periodontitis [6]. Other studies found that scaling and root planing resulted in a significant decrease in serum hsCRP and IL-6 up to 3 months posttreatment in otherwise healthy patients with periodontitis, while other inflammatory biomarkers were not affected [30, 33]; decrease in hsCRP and IL-6 in these studies also correlated with improvement in clinical parameters. Furthermore, pharmacologic anti-inflammatory therapy alone in patients with periodontitis was shown to result in significant decrease in serum hsCRP, IL-6, and IFN, while other inflammatory biomarkers remained unaffected [41]. In this study, however, there was no improvement in clinical parameters until mechanical therapy was initiated. On the other hand, Offenbacher et al. reported that in a multi-center study, there was no significant difference in serum hsCRP at 6 months between patients receiving scaling and root planing and patients receiving community care [35]. The authors noted, however, that there was great variability with regard to treatment provided in community care and that obesity was a confounding factor that nullified the periodontal treatment effects on hsCRP reduction. Based on the existing data, serum hsCRP and IL-6 are promising systemic biomarkers for monitoring the response to periodontal treatment in patients who are systemically healthy, non-smokers, and non-obese and do not take anti-inflammatory medications.

In a pilot intervention study, Duarte et al. evaluated the serum concentration of several cytokines in patients with generalized chronic periodontitis, generalized aggressive periodontitis and healthy controls, and the effect of periodontal therapy on these cytokines 6 months after treatment [9•]. They found that TNF-alpha and IL-17 concentrations were statistically significantly higher in aggressive periodontitis patients compared to healthy controls or chronic periodontitis, while there was no difference in other cytokines. In addition, nonsurgical periodontal therapy significantly decreased plasma TNF-alpha and IL-17 compared to baseline; both results were statistically significant; however, TNF-alpha levels remained elevated compared to healthy controls. These results suggest that serum TNF-alpha and IL-17 may be useful biomarkers for the differential diagnosis between aggressive and chronic periodontitis, while IL-17 may be useful for monitoring response to periodontal treatment in aggressive periodontitis.

Oncostatin M, a member of the IL-6 family of cytokines, is synthesized and secreted by T cells and monocytes in response to bacterial products; it has been shown to have a key role in regulating periodontal bone resorption, by acting on both osteoblast and osteoclast receptor activator of nuclear factor- $\mathrm{K} B$ ligand (RANKL) regulation [29]. Serum oncostatin M was significantly higher in patients with chronic periodontitis compared to healthy and gingivitis controls [38]. Furthermore, oncostatin $\mathrm{M}$ significantly decreased 2 months after nonsurgical periodontal therapy, and this decrease correlated with improvement in clinical parameters. These data suggest that serum oncostatin M may be a useful biomarker for the diagnosis as well as monitoring the response to periodontal treatment. 
In a cross-sectional and intervention study, Marcaccini et al. evaluated the plasma concentration of several MMPs and TIMPs in patients with chronic periodontitis and healthy controls, and the effect of periodontal therapy on these enzymes 3 months after treatment [31•]. They found that MMP-3, MMP-8, and MMP-9 concentrations were statistically significantly higher in periodontitis patients compared with healthy controls, while there was no difference in MMP-2, TIMP-1, and TIMP-2 levels. In addition, non-surgical periodontal therapy significantly decreased plasma MMP-8 and MMP-9 concentrations by 35 and $39 \%$ compared to baseline; both results were statistically significant. These results suggest that plasma MMP-3, MMP-8, and MMP-9 may be useful biomarkers for the diagnosis of diseased individuals, while MMP-8 and MMP-9 may be useful for monitoring response to periodontal treatment.

\section{Response to Treatment-Saliva Biomarkers}

The receptor activator of NF-KB (RANK) is found on the surface of osteoclasts; along with its agonist, receptor activator of NF-KB ligand (RANKL), and its antagonist, osteoprotegerin (OPG), they are the three key molecules important for coordinating osteoclastogenesis and alveolar bone resorption [43]. RANKL and OPG have been studied mainly in GCF samples and their concentrations have shown great variation from study to study. In a cross-sectional study comparing untreated patients with periodontitis to patients on periodontal maintenance, Buduneli et al. found that salivary RANKL levels were statistically significantly lower in maintenance non-smokers compared to untreated non-smokers [7]. When comparing maintenance smokers to untreated smokers, however, this difference was not significant, indicating that the effect of smoking on RANKL and OPG was such that these biomarkers could not be utilized to determine disease status in smokers. This data suggest that salivary RANKL may be a promising systemic biomarker for monitoring response to periodontal treatment in non-smokers but not in smokers.

CD44 is a cell-surface adhesion molecule that has been known to mediate cell-cell and cell-matrix interactions, and support of cell migration, including neutrophil adhesion and transendothelial migration [23, 48]. In a pilot intervention study evaluating the effect of scaling and root planing on salivary sCD44 levels, Ghallab and Shaker found that treatment resulted in significant decrease in SCD44 in patients with chronic periodontitis at 1 month after treatment [14]. Furthermore, this decrease was observed in both smokers and nonsmokers, suggesting that salivary sCD44 may be a promising systemic biomarker for monitoring response to periodontal treatment in both smokers and non-smokers.

Arginase is the final enzyme in the urea cycle that converts L-arginine to urea and L-ornithine, and although it is mainly found in the human liver, it is also present in some extrahepatic tissues, including salivary glands. Nitric oxide is a free radical with antimicrobial activity that has been shown to be produced in response to periodontal pathogens [3]. Nitric oxide is produced by L-arginine under the action of an isoenzyme called NOS. Considering that L-arginine is a common substrate for both arginase and NOS, it is believed that an increase in salivary arginase activity would lead to a decrease in the production of nitric oxide, potentially reducing its antimicrobial effect in the oral cavity and increasing susceptibility to periodontal disease. In a cross-sectional and intervention study, Gheren et al. evaluated the salivary arginase activity in patients with chronic periodontitis and healthy controls, and the effect of periodontal therapy on the activity of such enzyme 1 month after treatment [15]. They found that salivary arginase activity was approximately 2.5 times higher in chronic periodontitis subjects when compared to periodontally healthy controls and that periodontal therapy significantly reduced salivary arginase activity levels by 1.56 times compared to baseline; both results were statistically significant. Although salivary arginase activity overall correlated with clinical parameters and could distinguish between periodontitis patients and controls, there was no significant correlation between salivary arginase activity level and disease severity among patients with periodontitis. These results suggest that salivary arginase activity may be a useful biomarker for the diagnosis of diseased individuals, but not disease severity, as well as for monitoring response to periodontal treatment.

Aspartate aminotransferase (AST), alanine aminotransferase (ALT), and lactate dehydrogenase (LDH) are intracellular enzymes released from the damaged cells of periodontal tissues into the GCF and saliva. In an intervention study evaluating the effect of scaling and root planing on salivary AST, ALT, and LDH levels 1 month after treatment, Yoshie et al. found that treatment resulted in significant decrease in LDH in all patients with chronic periodontitis; AST and ALT were also significantly decreased in patients who were not carriers of the IL-1A allele, whereas no significant change was observed in the AST and ALT levels in IL-1A allele carriers [46]. All results for all enzymes were consistent between smokers and non-smokers. This data suggest that salivary LDH may be a promising systemic biomarker for monitoring response to periodontal treatment, and it is independent of smoking status and IL-1A allele status. In addition, salivary AST and ALT may be promising systemic biomarkers for monitoring response to periodontal treatment for patients who are IL-1A allele carriers, independent of their smoking status.

\section{Future Disease Prediction and Disease Progression Assessment-Blood Biomarkers}

Currently, the most reliable prognostic indicators for future attachment loss are bleeding on probing, especially when persistent $[19,26]$, and number of sites with probing depth of 
$5 \mathrm{~mm}$ or more [45]; none of these indicators demonstrate high sensitivity and specificity; however, they are the best indicators currently available. Thus, the biomarker with the highest potential for use in clinical practice is one that could accurately predict future disease progress or even identify currently active sites.

In a longitudinal study, Amarasena et al. followed 266 elderly Japanese yearly over 6 years in order to determine the correlation between periodontal disease progress and several systemic biomarkers, specifically serum calcium (Ca), albumin, random blood sugar, and immunoglobulin (IgG, IgA, and IgM) measured at baseline [4]. Progress of periodontal disease in this study was defined as the number of teeth that showed additional CAL loss of $3 \mathrm{~mm}$ or more. A multinomial logistic regression analysis determined that, of all biomarkers studied, serum $\mathrm{Ca}$ at baseline was the only variable that was significantly associated with periodontal disease progression over 6 years, with relative risks of 100-1000 depending on the degree of progression. This data suggest that serum Ca may be a useful systemic biomarker for predicting future progress of periodontal disease in the elderly. Additional studies are needed in order to determine if this biomarker can also be useful for other populations.

In a similar longitudinal study, Iwasaki et al. followed 304 elderly Japanese yearly over 4 years in order to determine the correlation between periodontal disease progress and several systemic biomarkers [21•]. A multiple regression analysis determined that, of all biomarkers studied, serum albumin at baseline, as well as over the years, was the only variable that was significantly associated with periodontal disease progress in non-smokers only, while this correlation was not observed in smokers. This data suggest that serum albumin may be a useful systemic biomarker for predicting future progress, as well as identifying progress, of periodontal disease in elderly non-smokers. Additional studies are needed in order to determine if this biomarker can also be useful for other populations.

\section{Conclusions}

The diagnosis of periodontitis is currently based almost entirely on an array of clinical measurements. The most reliable prognostic indicators available for future disease progression are also based on clinical measurements. These clinical measurements not only demonstrate low sensitivity and specificity as diagnostic tests, but are also subjective and laborious and require a lot of chair time. Reliable systemic biomarkers for periodontal disease could be very useful, if available as a diagnostic test; one could envisage chair-side tests using saliva or blood from finger sticks, or blood drawn for routine diagnostic check-ups, be used for the diagnosis and monitoring of periodontitis. Multiple local and systemic periodontal diagnostic biomarkers have been proposed within the literature; however, only one is presently commercially available in some European countries. Other tests are still at an early stage of development and much work remains to be performed to fully validate their utility such that they become an important and cost effective aspect of clinical diagnosis, treatment planning, or patient monitoring. The systemic biomarkers with the highest potential to be used as diagnostic and/or monitoring tests, along with their source, and specific populations they are applicable to, are summarized here and in Table 1.

Biomarkers with potential to be used for diagnosis of periodontal disease:

- $\quad$ Serum IgG levels to $P$. gingivalis may be useful systemic biomarkers for diagnosis of both chronic and aggressive periodontitis.

- Plasma surfactant protein -D may be a useful systemic biomarker for diagnosis of periodontitis.

- Commonly surveyed inflammatory and metabolic biomarkers may be promising systemic biomarkers for diagnosis of periodontitis in systemically healthy, nonsmokers Caucasians.

- Serum hsCRP and IL-6 may be useful systemic biomarkers for diagnosis of periodontitis.

- Serum cortisol may be a useful systemic biomarker for diagnosis of periodontitis in non-smokers.

- Serum osteocalcin may be a useful systemic biomarker for diagnosis of periodontitis in elderly Japanese.

- Serum oncostatin M may be a useful biomarker for the diagnosis of periodontitis.

- Salivary TNF-alpha may be a useful systemic biomarker for diagnosis of periodontitis.

- Plasma MMP-3, MMP-8, and -9 may be useful biomarkers for the diagnosis of periodontitis.

- Salivary MMP-8 and TIMP-1 are useful systemic biomarkers for diagnosis of periodontitis in non-smokers, while MMP-8/TIMP-1 ratio may be promising as a biomarker for smokers as well.

- Salivary IL-1beta and MMP-8 may be useful systemic biomarkers for diagnosis of periodontitis, and when evaluated in combination, they may increase the sensitivity of the diagnostic test.

- Combination of salivary MMP-8, MMP-9, and osteoprotegerin with red-complex anaerobic periodontal pathogens provided highly accurate predictions of periodontal disease category.

- Salivary arginase activity may be a useful biomarker for the diagnosis of periodontitis, but not disease severity.

Biomarkers with potential to be used for monitoring response to periodontal treatment: 
Table 1 Summary of biomarkers with the potential to be used for periodontitis diagnosis, treatment response monitoring, future disease prediction, and/or disease progression assessment. Biomarker source and specific population applicable identified

\begin{tabular}{|c|c|c|c|c|c|c|}
\hline Biomarker & Source & Diagnosis & $\begin{array}{l}\text { Treatment } \\
\text { response } \\
\text { monitoring }\end{array}$ & $\begin{array}{l}\text { Future } \\
\text { disease } \\
\text { prediction }\end{array}$ & $\begin{array}{l}\text { Disease } \\
\text { progression } \\
\text { assessment }\end{array}$ & Specific population \\
\hline IgG levels to $P$. gingivalis & Serum & $\begin{array}{l}\text { Chronic and aggressive } \\
\text { periodontitis }\end{array}$ & & & & \\
\hline Surfactant protein-D & Plasma & Periodontitis & & & & \\
\hline $\begin{array}{l}\text { Inflammatory and metabolic } \\
\text { biomarkers }\end{array}$ & Blood & Periodontitis & & & & $\begin{array}{l}\text { Caucasians, systemically healthy, } \\
\text { non-smokers }\end{array}$ \\
\hline hsCRP & Serum & Periodontitis & Periodontitis* & & & $\begin{array}{l}\text { * Systemically healthy, } \\
\text { non-smokers, non-obese, no } \\
\text { anti-inflammatory medications }\end{array}$ \\
\hline IL-6 & Serum & Periodontitis & Periodontitis* & & & $\begin{array}{l}\text { * Systemically healthy, non- } \\
\text { smokers, non-obese, no anti- } \\
\text { inflammatory medications }\end{array}$ \\
\hline Cortisol & Serum & Periodontitis & & & & Non-smokers \\
\hline Osteocalcin & Serum & Periodontitis & & & & Elderly Japanese \\
\hline Oncostatin M & Serum & Periodontitis & Periodontitis & & & \\
\hline TNF-alpha & Saliva & Periodontitis & & & & \\
\hline MMP-3 & Plasma & Periodontitis & & & & \\
\hline MMP-8 & Plasma & Periodontitis & Periodontitis & & & \\
\hline MMP-8 & Saliva & Periodontitis & & & & Non-smokers \\
\hline MMP-9 & Plasma & Periodontitis & Periodontitis & & & \\
\hline TIMP-1 & Saliva & Periodontitis & & & & Non-smokers \\
\hline MMP-8/TIMP-1 & Saliva & Periodontitis & & & & Smokers and non-smokers \\
\hline IL-1beta & Saliva & Periodontitis & & & & \\
\hline $\begin{array}{l}\text { MMP-8, MMP-9, } \\
\text { osteoprotegerin, red- } \\
\text { complex pathogens }\end{array}$ & Saliva & Periodontitis & & & & \\
\hline Arginase activity & Saliva & Periodontitis & Periodontitis & & & \\
\hline RANKL & Saliva & & Periodontitis & & & Non-smokers \\
\hline sCD44 & Saliva & & Periodontitis & & & Smokers and non-smokers \\
\hline LDH & Saliva & & Periodontitis & & & Smokers and non-smokers \\
\hline AST and ALT & Saliva & & Periodontitis & & & $\begin{array}{l}\text { IL-1A allele carriers, smokers } \\
\text { and non-smokers }\end{array}$ \\
\hline $\mathrm{Ca}$ & Serum & & & Periodontitis & & Elderly Japanese \\
\hline Albumin & Serum & & & Periodontitis & Periodontitis & Elderly Japanese, non-smokers \\
\hline
\end{tabular}

Asterisks indicate specific population studied for specific application

- Serum hsCRP and IL-6 are promising systemic biomarkers for monitoring the response to periodontal treatment in patients who are systemically healthy, nonsmokers, and non-obese and do not take antiinflammatory medications.

- Serum oncostatin M may be a useful biomarker for monitoring response to periodontal treatment.

- Plasma MMP-8 and MMP-9 may be useful for monitoring response to periodontal treatment.

- Salivary RANKL may be a promising systemic biomarker for monitoring response to periodontal treatment in nonsmokers.
- Salivary sCD44 may be a promising systemic biomarker for monitoring response to periodontal treatment in both smokers and non-smokers.

- Salivary arginase activity may be a useful biomarker for monitoring response to periodontal treatment.

- Salivary LDH may be a promising systemic biomarker for monitoring response to periodontal treatment, in both smokers and non-smokers.

- Salivary AST and ALT may be promising systemic biomarkers for monitoring response to periodontal treatment for patients who are IL-1A allele carriers, in both smokers and non-smokers. 
Biomarkers with potential to be used for future disease prediction and disease progression assessment:

- Serum Ca may be a useful systemic biomarker for predicting future progress of periodontal disease in elderly Japanese.

- Serum albumin may be a useful systemic biomarker for predicting future progress, as well as identifying progress, of periodontal disease in elderly Japanese non-smokers.

\section{Compliance with Ethical Standards}

Conflict of Interest Dr. Stathopoulou, Dr. Buduneli, and Dr. Kinane have nothing to disclose.

Human and Animal Rights and Informed Consent This article does not contain any studies with human or animal subjects performed by any of the authors.

\section{References}

Papers of particular interest, published recently, have been highlighted as:

- Of importance

1. American Academy of Periodontology Task Force Report on the Update to the 1999 Classification of Periodontal Diseases and Conditions. J Periodontol. 2015;86: 835-838. doi:10.1902/jop. 2015.157001.

2. Aas JA, Paster BJ, Stokes LN, Olsen I, Dewhirst FE. Defining the normal bacterial flora of the oral cavity. J Clin Microbiol. 2005;43: 5721-32. doi:10.1128/JCM.43.11.5721-5732.2005.

3. Akopov SE, Kankanian AP. Nitric oxide (NO) inactivation by polymorphonuclear leukocytes as a mechanism for the development of periodontal lesions. Stomatologiia (Mosk). 1996;75:12-4.

4. Amarasena N, Yoshihara A, Hirotomi T, Takano N, Miyazaki H. Association between serum calcium and periodontal disease progression in non-institutionalized elderly. Gerodontology. 2008;25: 245-50. doi:10.1111/j.1741-2358.2007.00211.x.

5. Armitage GC. Development of a classification system for periodontal diseases and conditions. Ann Periodontol. 1999;4:1-6. doi:10. 1902/annals.1999.4.1.1.

6. Behle JH, Sedaghatfar MH, Demmer RT, Wolf DL, Celenti R, Kebschull M, et al. Heterogeneity of systemic inflammatory responses to periodontal therapy. J Clin Periodontol. 2009;36:28794. doi:10.1111/j.1600-051X.2009.01382.x.

7. Buduneli N, Biyikoglu B, Sherrabeh S, Lappin DF. Saliva concentrations of RANKL and osteoprotegerin in smoker versus nonsmoker chronic periodontitis patients. J Clin Periodontol. 2008;35:846-52. doi:10.1111/j.1600-051X.2008.01310.x.

8. Craig RG, Yip JK, So MK, Boylan RJ, Socransky SS, Haffajee AD. Relationship of destructive periodontal disease to the acute-phase response. J Periodontol. 2003;74:1007-16. doi:10.1902/jop.2003. 74.7.1007.

9. Duarte PM, da Rocha M, Sampaio E, Mestnik MJ, Feres M, Figueiredo LC, et al. Serum levels of cytokines in subjects with generalized chronic and aggressive periodontitis before and after non-surgical periodontal therapy: a pilot study. J Periodontol. 2010;81:1056-63. doi:10.1902/jop.2010.090732. This is the only study that provides evidence of potential systemic biomarkers that can be promising for the differential diagnosis between aggressive and chronic periodontitis.

10. Ebersole JL, Machen RL, Steffen MJ, Willmann DE. Systemic acute-phase reactants, C-reactive protein and haptoglobin, in adult periodontitis. Clin Exp Immunol. 1997;107:347-52.

11. Eke PI, Dye BA, Wei L, Thornton-Evans GO, Genco RJ, Cdc Periodontal Disease Surveillance workgroup: James Beck, G. D. R. P. (2012) Prevalence of periodontitis in adults in the United States: 2009 and 2010. J Dent Res 91, 914-920. doi:10.1177/ 0022034512457373.

12. Fredriksson MI, Figueredo CM, Gustafsson A, Bergstrom KG, Asman BE. Effect of periodontitis and smoking on blood leukocytes and acute-phase proteins. J Periodontol. 1999;70:1355-60. doi:10.1902/jop.1999.70.11.1355.

13. Frodge BD, Ebersole JL, Kryscio RJ, Thomas MV, Miller CS. Bone remodeling biomarkers of periodontal disease in saliva. J Periodontol. 2008;79:1913-9. doi:10.1902/jop.2008.080070.

14. Ghallab N, Shaker O. Salivary-soluble CD44 levels in smokers and non-smokers with chronic periodontitis: a pilot study. J Periodontol. 2010;81:710-7. doi:10.1902/jop.2010.090630.

15. Gheren LW, Cortelli JR, Rodrigues E, Holzhausen M, Saad WA. Periodontal therapy reduces arginase activity in saliva of patients with chronic periodontitis. Clin Oral Investig. 2008;12:67-72. doi: 10.1007/s00784-007-0146-8.

16. Glas J, Beynon V, Bachstein B, Steckenbiller J, Manolis V, Euba A, et al. Increased plasma concentration of surfactant protein D in chronic periodontitis independent of SFTPD genotype: potential role as a biomarker. Tissue Antigens. 2008;72:21-8. doi:10.1111/ j.1399-0039.2008.01056.x.

17. Glurich I, Grossi S, Albini B, Ho A, Shah R, Zeid M, et al. Systemic inflammation in cardiovascular and periodontal disease: comparative study. Clin Diagn Lab Immunol. 2002;9:425-32.

18. Gursoy UK, Kononen E, Pradhan-Palikhe P, Tervahartiala T, Pussinen PJ, Suominen-Taipale L, et al. Salivary MMP-8, TIMP1 , and ICTP as markers of advanced periodontitis. J Clin Periodontol. 2010;37:487-93. doi:10.1111/j.1600-051X.2010. 01563.x.

19. Haffajee AD, Socransky SS, Goodson JM. Clinical parameters as predictors of destructive periodontal disease activity. J Clin Periodontol. 1983;10:257-65.

20. Ishisaka A, Ansai T, Soh I, Inenaga K, Awano S, Yoshida A, et al. Association of cortisol and dehydroepiandrosterone sulphate levels in serum with periodontal status in older Japanese adults. J Clin Periodontol. 2008;35:853-61. doi:10.1111/j.1600-051X.2008. 01309.x.

21. Iwasaki M, Yoshihara A, Hirotomi T, Ogawa H, Hanada N, Miyazaki H. Longitudinal study on the relationship between serum albumin and periodontal disease. J Clin Periodontol. 2008;35:2916. doi:10.1111/j.1600-051X.2008.01215.x. This is the only study that provides evidence of potential systemic biomarkers that can be promising for predicting future progress, as well as identifying progress, of periodontal disease.

22. Kaufman E, Lamster IB. Analysis of saliva for periodontal diagnosis - a review. J Clin Periodontol. 2000;27:453-65.

23. Khan AI, Kerfoot SM, Heit B, Liu L, Andonegui G, Ruffell B, et al. Role of CD44 and hyaluronan in neutrophil recruitment. J Immunol. 2004;173:7594-601.

24. Kinane DF, Attstrom R, European Workshop in Periodontology group, B. (2005) Advances in the pathogenesis of periodontitis. Group B consensus report of the fifth European Workshop in Periodontology. J Clin Periodontol; 32 Suppl 6, 130-131. doi:10. 1111/j.1600-051X.2005.00823.x. 
25. Kinane DF, Peterson M, Stathopoulou PG. Environmental and other modifying factors of the periodontal diseases. Periodontol. 2006;2000(40):107-19. doi:10.1111/j.1600-0757.2005.00136.x.

26. Lang NP, Joss A, Orsanic T, Gusberti FA, Siegrist BE. Bleeding on probing. A predictor for the progression of periodontal disease? J Clin Periodontol. 1986;13:590-6.

27. Liu J, Wu Y, Ding Y, Meng S, Ge S, Deng H. Evaluation of serum levels of C-reactive protein and lipid profiles in patients with chronic periodontitis and/or coronary heart disease in an ethnic Han population. Quintessence Int. 2010;41:239-47.

28. Loos BG, Craandijk J, Hoek FJ, Wertheim-van Dillen PM, van der Velden U. Elevation of systemic markers related to cardiovascular diseases in the peripheral blood of periodontitis patients. J Periodontol. 2000;71:1528-34. doi:10.1902/jop.2000.71.10.1528.

29. Lu HK, Chen YL, Chang HC, Li CL, Kuo MY. Identification of the osteoprotegerin/receptor activator of nuclear factor-kappa B ligand system in gingival crevicular fluid and tissue of patients with chronic periodontitis. J Periodontal Res. 2006;41:354-60. doi:10.1111/j. 1600-0765.2006.00883.x.

30. Marcaccini AM, Meschiari CA, Sorgi CA, Saraiva MC, de Souza AM, Faccioli LH, et al. Circulating interleukin-6 and highsensitivity C-reactive protein decrease after periodontal therapy in otherwise healthy subjects. J Periodontol. 2009;80:594-602. doi: 10.1902/jop.2009.080561.

31. Marcaccini AM, Novaes Jr AB, Meschiari CA, Souza SL, Palioto DB, Sorgi CA, et al. Circulating matrix metalloproteinase-8 (MMP8) and MMP-9 are increased in chronic periodontal disease and decrease after non-surgical periodontal therapy. Clin Chim Acta. 2009;409:117-22. doi:10.1016/j.cca.2009.09.012. This study evaluated a wide range of MMPs and TIMPs, which have a well-documented role in periodontitis, and identified a panel of MMPs that can be promising for the diagnosis of periodontitis as well as monitoring response to periodontal treatment.

32. Miller CS, King Jr CP, Langub MC, Kryscio RJ, Thomas MV. Salivary biomarkers of existing periodontal disease: a crosssectional study. J Am Dent Assoc. 2006;137:322-9.

33. Nakajima T, Honda T, Domon H, Okui T, Kajita K, Ito H, et al. Periodontitis-associated up-regulation of systemic inflammatory mediator level may increase the risk of coronary heart disease. J Periodontal Res. 2010;45:116-22. doi:10.1111/j.1600-0765.2009. 01209.x.

34. Nibali L, D'Aiuto F, Griffiths G, Patel K, Suvan J, Tonetti MS. Severe periodontitis is associated with systemic inflammation and a dysmetabolic status: a case-control study. J Clin Periodontol. 2007;34:931-7. doi:10.1111/j.1600-051X.2007.01133.x.

35. Offenbacher S, Beck JD, Moss K, Mendoza L, Paquette DW, Barrow DA, et al. Results from the Periodontitis and Vascular Events (PAVE) Study: a pilot multicentered, randomized, controlled trial to study effects of periodontal therapy in a secondary prevention model of cardiovascular disease. J Periodontol. 2009;80:190-201. doi:10.1902/jop.2009.080007.
36. Ozcaka O, Nalbantsoy A, Kose T, Buduneli N. Plasma osteoprotegerin levels are decreased in smoker chronic periodontitis patients. Aust Dent J. 2010;55:405-10. doi:10.1111/j.1834-7819. 2010.01261.x.

37. Paster BJ, Boches SK, Galvin JL, Ericson RE, Lau CN, Levanos VA, et al. Bacterial diversity in human subgingival plaque. J Bacteriol. 2001;183:3770-83. doi:10.1128/JB.183.12.3770-3783. 2001.

38. Pradeep AR, S TM, Garima G, Raju A. Serum levels of oncostatin M (a gp 130 cytokine): an inflammatory biomarker in periodontal disease. Biomarkers. 2010;15: 277-282. doi:10.3109/ 13547500903573209.

39. Rai B, Kharb S, Jain R, Anand SC. Biomarkers of periodontitis in oral fluids. J Oral Sci. 2008;50:53-6.

40. Ramseier CA, Kinney JS, Herr AE, Braun T, Sugai JV, Shelburne $\mathrm{CA}$, et al. Identification of pathogen and host-response markers correlated with periodontal disease. J Periodontol. 2009;80:43646. doi:10.1902/jop.2009.080480.

41. Renvert S, Lindahl C, Roos-Jansaker AM, Lessem J. Short-term effects of an anti-inflammatory treatment on clinical parameters and serum levels of C-reactive protein and proinflammatory cytokines in subjects with periodontitis. J Periodontol. 2009;80:892900. doi:10.1902/jop.2009.080552.

42. Sorsa T, Tjaderhane L, Salo T. Matrix metalloproteinases (MMPs) in oral diseases. Oral Dis. 2004;10:311-8. doi:10.1111/j.16010825.2004.01038.x.

43. Suda T, Takahashi N, Udagawa N, Jimi E, Gillespie MT, Martin TJ. Modulation of osteoclast differentiation and function by the new members of the tumor necrosis factor receptor and ligand families. Endocr Rev. 1999;20:345-57. doi:10.1210/edrv.20.3.0367.

44. Trindade SC, Gomes-Filho IS, Meyer RJ, Vale VC, Pugliese L, Freire SM. Serum antibody levels against Porphyromonas gingivalis extract and its chromatographic fraction in chronic and aggressive periodontitis. J Int Acad Periodontol. 2008;10:50-8.

45. Van der Velden U, Abbas F, Armand S, Loos BG, Timmerman MF, Van der Weijden GA, et al. Java project on periodontal diseases. The natural development of periodontitis: risk factors, risk predictors and risk determinants. J Clin Periodontol. 2006;33:540-8. doi: 10.1111/j.1600-051X.2006.00953.x.

46. Yoshie H, Tai H, Kobayashi T, Oda-Gou E, Nomura Y, Numabe Y, et al. Salivary enzyme levels after scaling and interleukin-1 genotypes in Japanese patients with chronic periodontitis. J Periodontol. 2007;78:498-503. doi:10.1902/jop.2007.060216.

47. Yoshihara A, Deguchi T, Hanada N, Miyazaki H. Relation of bone turnover markers to periodontal disease and jaw bone morphology in elderly Japanese subjects. Oral Dis. 2009;15:176-81. doi:10. 1111/j.1601-0825.2008.01511.x.

48. Zhuo L, Kanamori A, Kannagi R, Itano N, Wu J, Hamaguchi M, et al. SHAP potentiates the CD44-mediated leukocyte adhesion to the hyaluronan substratum. J Biol Chem. 2006;281:20303-14. doi: 10.1074/jbc.M506703200. 